\title{
Atrial structure in the presence of visceral heterotaxy
}

\author{
Robert H. Anderson \\ Cardiac Unit, Institute of Child Health, University College, London, UK
}

$\mathrm{T}$ HE STRUCTURE OF THE HEART IN THE SETTING of so-called visceral heterotaxy remains a potentially controversial subject. Although the existence of an isomeric arrangement of the lungs and bronchial tree in patients with "splenic syndromes" was long since established, ${ }^{1}$ there are still acknowledged experts who deny that the heart itself shows any evidence of isomerism. ${ }^{2}$ The study published in this issue of the Journal ${ }^{3}$ goes a long way to clarifying the bones of contention, confirming not only that the appendages themselves are unequivocally isomeric, but that the venous components of the atrial chambers are similarly arranged to reflect the presence of either bilateral right sidedness or left sidedness. The study shows the value of analysing congenitally malformed atrial chambers in terms of their appendages and venous components, but emphasises the need also to recognise the body of the atrial segment, from which the appendages and the septum grow during embryological development. It is this segment which persists in the formed heart as the vestibules of the atrioventricular junctions, but in addition forms a significant portion of the definitive morphologically left atrium. In this note, I will review briefly the background to these morphological and developmental issues.

\section{Morphological analysis of visceral heterotaxy}

It is now well recognised that the normal arrangement of the body is one of asymmetry, with the right side being morphologically distinct from the left, as we discussed in our recent review published in the Journal. ${ }^{4}$ Thus, in addition to the usual

Correspondence to. Professor Robert H. Anderson, Cardiac Unit, Institute of Child Health, University College London, 30 Guldford Street, London WCIN IEH, UK, Tel: (020) 7905 2322; Fax: (020) 79052324

Accepted for publication 3 May 2000 arrangement of the organs within the body, and the rare mirror-imaged variant, often called "situs inversus", there is a commoner abnormal pattern in which the abdominal organs are "jumbled up". It is this third group, with visceral heterotaxy, which creates problems not only in terms of diagnosis but also in description. All the disputes, nonetheless, are clarified by application of the "Morphological method".

This principle of anatomic analysis was first used by Van Praagh and his colleagues ${ }^{5}$ as a justifiable criticism of our initial approach to the "univentricular heart". ${ }^{6}$ At that time, we had promulgated the concept that, so as to make logical the description of "single ventricle" in hearts with two ventricular chambers of dissimilar size and component makeup, we should disqualify chambers from ventricular status when they lacked their inlet components. Van Praagh et al. ${ }^{5}$ highlighted the conceptual deficiencies produced when one variable feature was itself defined in terms of another feature which was also variable. Instead, they argued, anatomical structures should be defined according to their intrinsic morphology, using their most constant component for the purposes of definition when the structures themselves were congenitally malformed. As a direct extension of this morphological method, it follows that, ideally, one system of organs should not be identified, or named, on the basis of abnormalities in another system. In fact, use of this method clarifies all of the apparent controversies which surround the subject of visceral heterotaxy.

It is agreed that the arrangement of the organs in this setting is far from normal. Indeed, in the past the unusual arrangements were often considered to be, and described as, "ambiguus". When each system is described in its own right, however, all aspects of potential ambiguity are removed. ${ }^{7}$ Analysis of large numbers of patients show that there are two subsets within the overall grouping of 
visceral heterotaxy. In the abdomens of these patients, the most characteristic feature is either absence of the spleen or the presence of multiple spleens. Within the thorax, in contrast, it is almost always an isomeric arrangement of the lungs and bronchial tree that dominates the picture. In the heart, when the cardiac structures are analysed within the precepts of the morphological method, then there is unequivocal evidence of isomerism of the atrial appendages. ${ }^{8}$ Usually, the presence of isomerism of the morphologically right atrial appendages is accompanied by right pulmonary and bronchial isomerism, and by absence of the spleen. Similarly, isomeric left atrial appendages are most usually found when the lungs and bronchuses on both sides are similarly of left morphology, and there are multiple spleens within the abdomen. But this is not always the case. Should there be discrepancies in the positions of the organs, then each system should be described separately and specifically. Within the heart, it is the isomeric arrangement of the atrial appendages, judged on the extent of the pectinate muscles relative to the atrioventricular junctions, which is the best discriminating feature when assessed in the setting of large numbers of hearts, with the venoatrial connections revealing marked variability. ${ }^{8}$ As the careful study from $\mathrm{Korea}^{3}$ now shows us, however, there are patterns to be found in the arrangement of the venous connections which reflect the presence of either bilateral right or left sidedness. The most obvious feature of the normal morphologically left atrium is the connection to it of the four pulmonary veins. But, following the rules of the morphological method, we are not permitted to use the pulmonary veins as the final arbiter of which chamber is morphologically left, since the chamber is readily recognised on the basis of its own intrinsic morphology even when the pulmonary veins connect to an extracardiac site, as in hearts with totally anomalous pulmonary venous connection (Fig. 1). Analysis of the left atrium in such hearts demonstrates the significance of the smooth-walled body of the left atrium, which gives rise to the characteristic appendage, supports the vestibule of the mitral valve, and is separated by the septum from the morphologically right atrium. In similar fashion, whilst the connections of the caval veins, particularly the inferior caval vein, are obvious guides to the existence of the morphologically right atrium, they cannot be used specifically to define that chamber. Indeed, the morphologically right atrium is indistinguishable from the normal chamber in those persons who have usual atrial arrangement and interruption of the inferior caval vein, with return of the blood from the abdomen

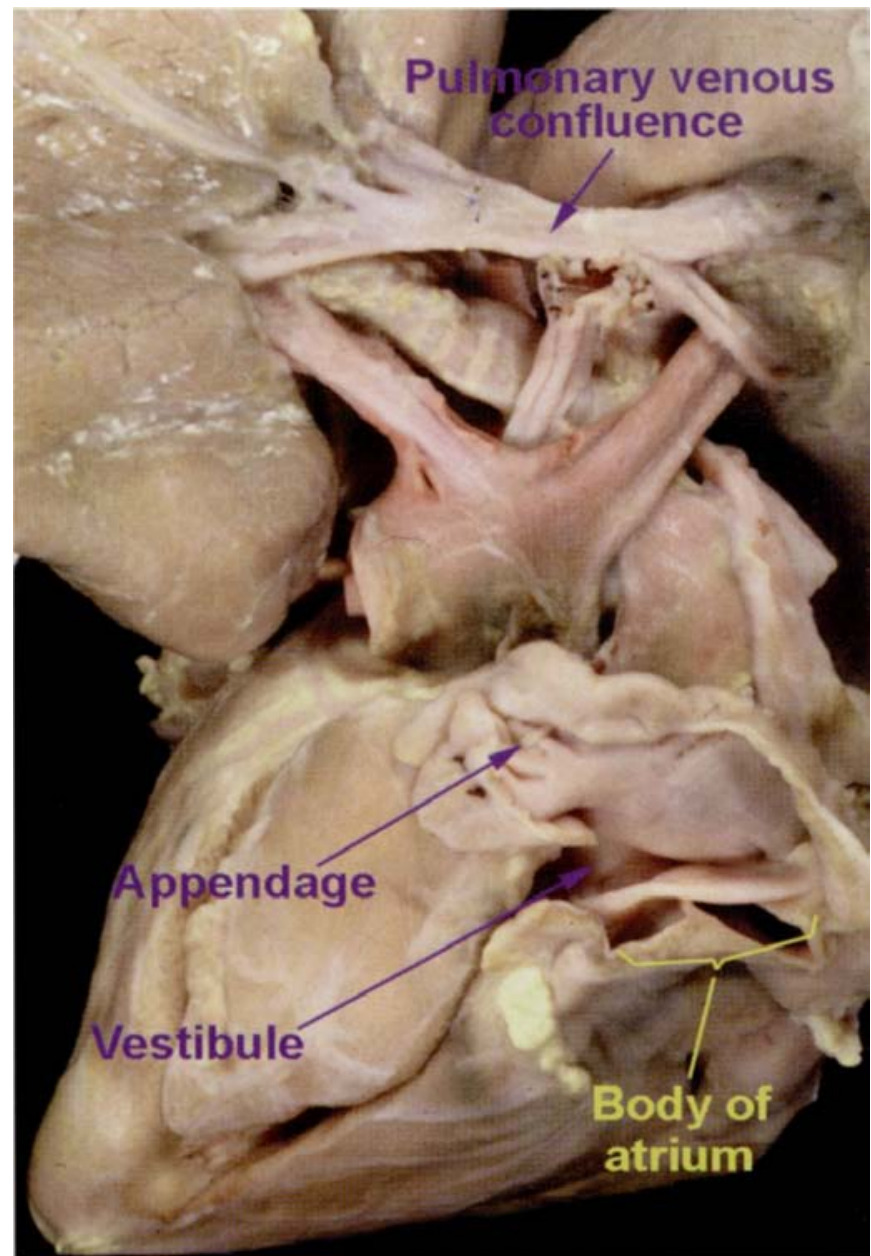

Figure 1.

This heart bad totally anomalous pulmonary venous connection, with the venous confluence initially draining infradiapbragmatically. As can be seen, the left atrium is recognised as such on the basis of the structure of its appendage. In addition to the vestzbule and the septum, nonetbeless, the atrum possesses a substantial smooth-walled body even in the absence of tts pulmonary venous component.

through the azygos venous system. As indicated, therefore, when following the morphological method, it is the atrial appendages that must be used to define the identity of the atrial chambers. For total clarity of description in the setting of visceral heterotaxy, nonetheless, it is essential to specify all the venoatrial connections. As the accompanying study shows, ${ }^{3}$ there are patterns which can then be anticipated in the setting of isomerism. When both appendages are of right morphology, then the venous components of the atrial segment similarly show the patterns of bilateral right sidedness, with preservation of an extensive smooth-walled systemic venous component on both sides, with universal failure during development of conversion of the morphologically left sinus horn into the coronary sinus. There is either total 
absence, or marked diminution in size, of the pulmonary venous contribution to the atriums. When connected to the heart, the pulmonary veins almost always enter the smooth-walled atrial component close to the mid-line. In contrast, in the presence of bilateral appendages of left morphology, there is far greater development of the pulmonary venous component, which is itself typically present bilaterally, increasing the size of the smooth-walled atrial component, but with diminution in size and importance of the systemic venous component, usually but not always with return of the inferior caval venous blood through the azygos system. The patterns of venous connection, nonetheless, continued to show variation in the hearts examined, ${ }^{3}$ confirming that it is the appendages themselves, with their contained pectinate muscles, which serve as the most consistent marker to the presence of isomerism in the atrial segment of the heart.

\section{Developmental implications}

The study from Korea ${ }^{3}$ is also important for those who continue to seek the molecular mechanisms which underscore the development of symmetry as opposed to lateralisation within the bodily organs. The evidence is becoming increasingly more convincing that concepts of the five-segmented primary heart tube are unduly simplistic. When the heart tube is first formed, the convincing studies of de la Cruz and her colleagues show that, in effect, it is made up of only the ventricular segments. ${ }^{9}$ These segments are themselves formed in series, and give rise to the apical components of the left and right ventricles, respectively. Although it is true that "looping" of this tube to either the right or left is an initial indication of the breaking of symmetry, ${ }^{10}$ it is equally true that formation of the ventricles in either a right hand or left hand loop has very little to do with subsequent isomeric development within the heart. Indeed, I am aware of only one well-documented example of ventricular isomerism. ${ }^{11}$ It is the atrial segment of the developing heart which needs to be the focus of those who seek the genes which govern the regulation of cardiac symmetry and asymmetry. As de la Cruz and her colleagues have shown," the atrial segment is younger in developmental terms than the ventricles. When first seen in the developing heart, however, it has an obvious body, into which the systemic venous tributaries initially drain in symmetrical fashion (Fig. 2). At the stage at which the body of the atrial segment is itself already well established, the lungs have yet to develop, and the atrial myocardium is continuous posteriorly with the substance of the developing

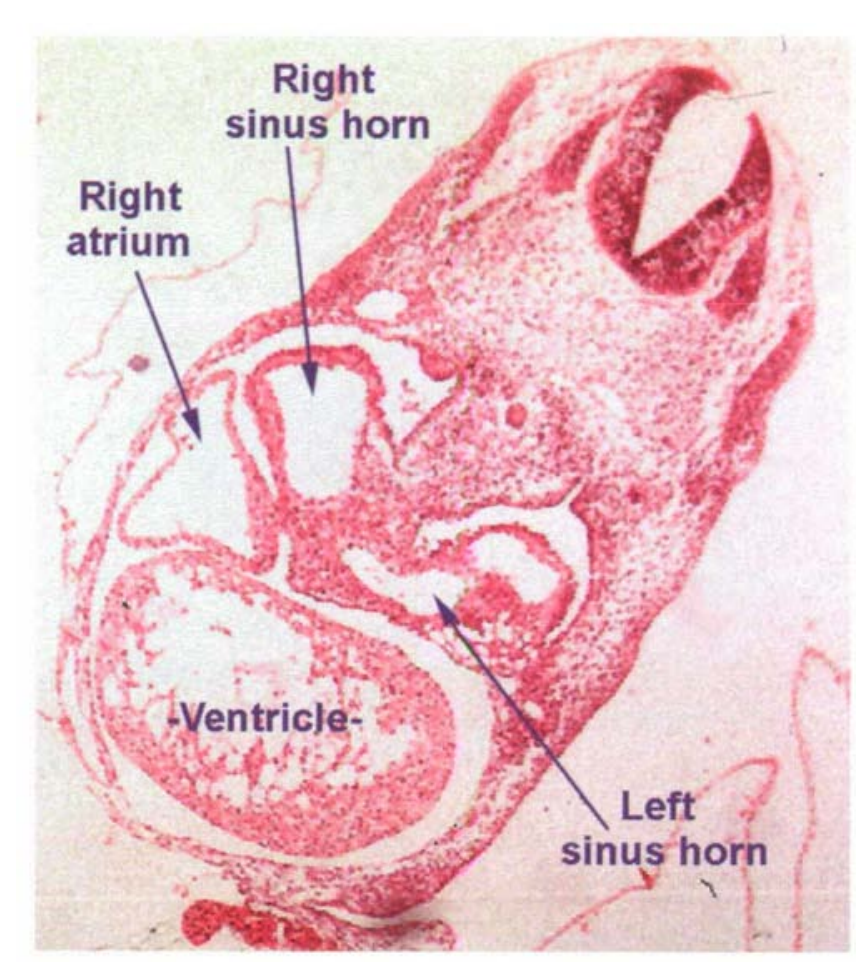

Figure 2.

This transverse section is from a $4 \mathrm{~mm}$ buman embryo obtained and sectioned by $\mathrm{Dr}$ Leon Gerlis, and reproduced with his permission. It shows the initial symmetry of the systemic venous tributaries, which drain from the outset into the body of the developing atrial segment (see Fig. 3).

mediastinum through the so-called "dorsal mesocardium" (Fig. 3). By this stage, nonetheless, the atrial appendages have already grown out in parallel from the body of this initial atrial segment. And, in the normally developing heart, each appendage can be recognised on the basis of its own intrinsic morphology from the first stage at which it is seen, negating suggestions that their shape reflects haemodynamic patterning. ${ }^{2}$ This normal pattern of development, with the body of the primary atrial segment giving rise to the appendages and vestibules of both definitive atriums, and also providing the scaffold for formation of the primary atrial septum, and then persisting as the body of the morphologically left atrium, provides the background for understanding the abnormal patterns described by the Korean group in hearts with isomeric appendages. ${ }^{3}$ The acquisition of the systemic venous component by the morphologically right atrium, and the appearance of the pulmonary vein in the dorsal mesocardium, with subsequent incorporation into the morphologically left atrium, are very much later events in development. ${ }^{12}$ Such incorporations are perturbed when the atrial segment itself is formed in symmetrical rather than lateralised pattern, as 


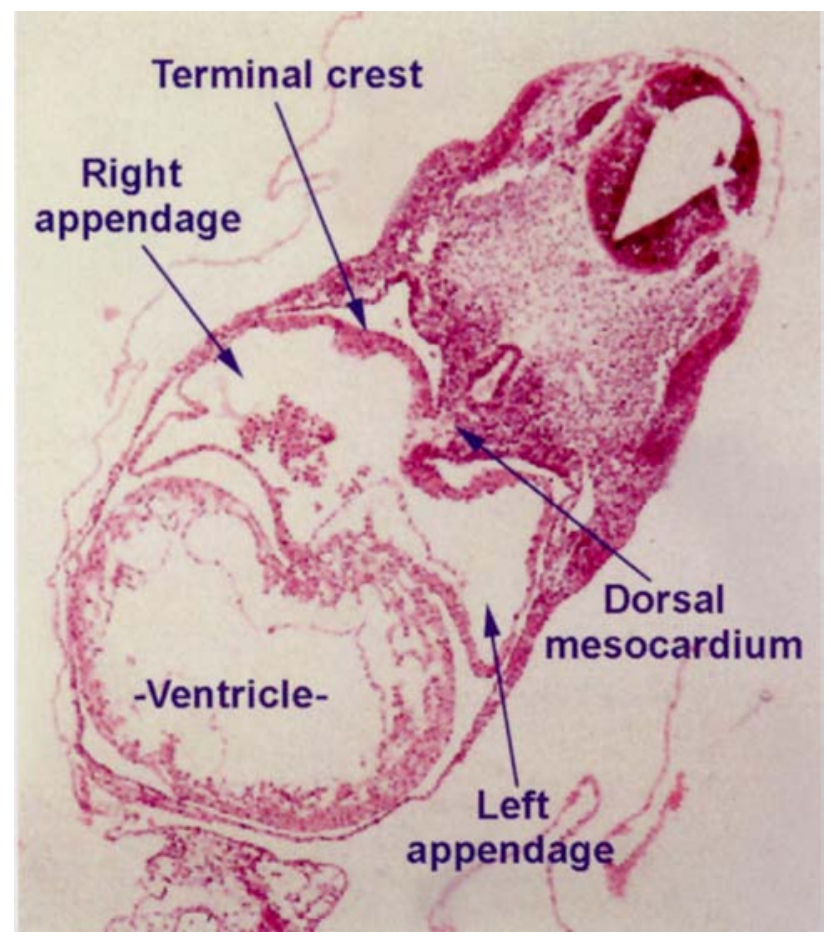

Figure 3.

This further section from the Gerlis $4 \mathrm{~mm}$ embryo, taken at a more cranial level than Figure 2, shows bow the body of the atrial segment is continuous posteriorly through myocardial-mesenchymal reflections with the tissues of the mediastinum - the so-called dorsal mesocardium. At this stage, neitber the lung buds nor the pulmonary vein bave yet developed. Already, bowever, the appendages have grown out from either side of the primary atrial segment, and are readily distingussed on the basis of their morphology.

shown by the structure of hearts with isomeric appendages. This atrial segment, and the developing atrioventricular junctions, ${ }^{4}$ therefore, need to be the focus if we are to clarify the developmental mechanisms leading to the formation of isomeric appendages, and their subsequent influence on the remainder of the heart. The mechanisms underscoring formation of the ventricular loop are also important, but these are more likely to form corollary events in the developmental cascade producing cardiac symmetry. As the Korean group have now shown, ${ }^{3}$ this involves the entirety of the atrial segment, albeit to greater or lesser degree.

\section{Acknowledgement}

I am grateful to Dr Leon Gerlis, Visiting Professor at the National Heart and Lung Institute, Imperial College, London, for granting me access to his personally prepared series of human embryos. I continue to be indebted to the British Heart Foundation, together with the Joseph Levy Foundation, for their ongoing support.

\section{References}

1. Landıng BH, Lawrence TK, Payne VC, Wells TR. Bronchial anatomy in syndromes with abnormal visceral situs, abnormal spleen and congenital heart disease. Am J Cardiol 1971;12: 456-462.

2. Van Praagh R, Van Praagh S. Atrial isomerism in the heterotaxy syndromes with asplenia, or polysplenia, or normally formed spleen: an erroneous concept. Am J Cardiol 1990;66: 1504-1506.

3. Min J-Y, Kim C-Y, Oh MH, Chun YK, Suh Y-L, Kang I-S, Lee $\mathrm{H}-\mathrm{J}$, Seo J-W. The arrangement of the systemic and pulmonary venous components of the atrial chambers in hearts with isomeric atrial appendages. Cardiol Young 2000;10:

4. Anderson RH, Webb S, Brown NA. Defective lateralisation in children with congenitally malformed hearts. Cardiol Young 1998;8: 512-531.

5. Van Praagh R, David I, Wright GB, Van Praagh S. Large RV plus small LV is not single ventricle. Circulation 1980,61: $1057-1058$

6. Keeton BR, Macartney FJ, Hunter S, Mortera C, Rees P, Shinebourne EA, Tynan M, Wilkinson JL, Anderson RH. Univentricular heart of right ventricular type with double or common inlet. Circulation 1979;59: 403-411.

7. Uemura H, Ho SY, Devine WA, Anderson RH. Analysis of visceral heterotaxy according to splenic status, appendage morphology, or both. Am J Cardiol 1995;76: 846-849.

8. Uemura H, Ho SY, Devine WA, Kilpatrick LL, Anderson RH Atrial appendages and venoatrial connections in hearts with patients with visceral heterotaxy. Ann Thorac Surg. 1995;60: 561-569

9. de la Cruz MV, Sanchez-Gomez C, Palomino MA. The primitive cardiac regions in the straight tube heart (Stage 9-) and their anatomical expression in the mature heart: an exper1mental study in the chick embryo. J Anat 1989;165: $121-131$.

10. Yost HJ. Inhibition of proteoglycan synthesis eliminates leftright asymmetry in Xenopus laevis cardiac looping Development 1990;110: 865-874.

11. Rinne K, Smith A, Ho SY. A unique case of ventricular isomerism? Cardiol Young 200;10:42-45

12. Webb S, Brown NA, Wessels A, Anderson RH. Development of the murine pulmonary vein and its relationship to the embryonic venous sinus. Anat Rec 1998;250: 325-334. 